\title{
2,4-Bis(1,1-dimethylethyl)phenol from Cinnamomum loureirii Improves Cognitive Deficit, Cholinergic Dysfunction, and Oxidative Damage in TMT-Treated Mice
}

\author{
Cho Rong Kim, ${ }^{a}{ }^{\dagger}$ Soo Jung Choi,${ }^{b}$ Jae Kyeom Kim, ${ }^{c}$ Chan Kyu Park, ${ }^{a}$ Min Chul Gim, ${ }^{a}$ \\ Youn-Jung Kim, ${ }^{d}$ Gwi Gun Park, ${ }^{e}$ and Dong-Hoon Shin*,a \\ ${ }^{a}$ Department of Food and Biotechnology, Korea University; Seoul 136-701, Korea: ${ }^{b}$ Functional Food Research \\ Center, Korea University; Seoul 136-701, Korea: ${ }^{c}$ School of Human Environmental Sciences, University of Arkansas; \\ Fayetteville, AR 72701, U.S.A.: ${ }^{d}$ College of Nursing Science, Kyunghee University; Seoul 136-701, Korea: and \\ ${ }^{e}$ Department of Food Science and Biotechnology, Gachon University; Seongnam 461-701, Korea. \\ Received December 19, 2016; accepted March 17, 2017
}

We previously reported that the extract of Cinnamomum loureirii (C. loureirii) significantly inhibited acetylcholinesterase (AChE), and identified 2,4-bis(1,1-dimethylethyl)phenol (BP) from C. loureirii as a potential AChE inhibitor. The present study, therefore was undertaken to demonstrate the effects of BP from C. loureirii on learning and memory impairment in trimethyltin (TMT)-treated ICR mice. Y-maze and passive avoidance tests were used to test cognitive ability. Further, changes in biochemical parameters in the brain tissue were also assessed in response to TMT injection and BP intervention. BP pre-administration $(20,40 \mathrm{mg} / \mathrm{kg} / \mathrm{d})$ in mice significantly protected cognitive dysfunction induced by TMT $(\boldsymbol{p}<0.05)$. Moreover, $\mathrm{BP}$ reduced $\mathrm{AChE}$ activity and lipid peroxidation but increased acetylcholine levels in the brain. In conclusion, we suggested that BP protected against TMT-induced cognitive dysfunction, and might be a potential agent for alleviating symptoms of neurodegenerative disorders, such as Alzheimer's disease, via modulating cholinergic functions.

Key words Alzheimer's disease; acetylcholinesterase inhibitor; Cinnamomum loureirii; 2,4-bis(1,1-dimethylethyl)phenol; cognitive dysfunction; trimethyltin

Alzheimer's disease (AD), a progressive neurodegenerative disorder, is the most common form of dementia in the elderly. The major pathological characteristics of AD include senile plaques, neurofibrillary tangles, and synapse loss. ${ }^{1)}$ The cholinergic system is involved in central cognitive processes, such as learning and memory. ${ }^{2)}$ And a deficiency of the neurotransmitter acetylcholine (ACh) is closely related with the severity of cognitive dysfunction in AD. ${ }^{3)}$ One of the major therapeutic strategies is, therefore the enhancement of ACh levels in the brain by the inhibition of the catabolic enzyme acetylcholinesterase (AChE). ${ }^{4}$

Traditionally, plants are a rich source of AChE inhibitors, and plant-derived drugs (e.g., galantamine isolated from daffodil bulbs) are used for the symptomatic treatment of AD. ${ }^{5}$ However, these drugs have known limitations owing to their side effects, and many researchers have focused on other natural sources of newer, potent, and long-lasting compounds with anti-AChE activity. ${ }^{6}$ ) As part of such efforts, we previously demonstrated that $C$. loureirii extract significantly inhibited $\mathrm{AChE}$ in vitro and was neuroprotective against trimethyltin (TMT)-induced cognitive dysfunction in mice. In addition, we identified 2,4-bis(1,1-dimethylethyl)phenol (BP) as the potential AChE inhibitor in C. loureirii extract.")

$\mathrm{BP}$ is a phenolic compound present in various plants. ${ }^{8,9)}$ The compound has been suggested to represent antioxidant, antibacterial, and antifungal activities. ${ }^{10-12)}$ To the best of our knowledge, there are no available reports on the effects of BP against TMT-induced cognitive impairment in the context of

†Present address: 612 R\&D Center, Korea University; 145 Anam-ro, Seongbuk-gu, Seoul 136-701, Republic of Korea.
AChE inhibition. The aim of the present work was therefore to investigate the possible cognitive protection of BP against neurodegeneration induced by TMT. In addition to mice behavioral tests, we further analyzed ex vivo AChE activity, ACh levels, and malondialdehyde (MDA) levels in the brain tissue of amnesic mice.

\section{MATERIALS AND METHODS}

Materials Acetylthiocholine iodide, 5,5'-dithiobis(2-nitro)benzoic acid (DTNB), BP, TMT chloride, and MDA were purchased from Sigma (St. Louis, MO, U.S.A.). All other chemicals used were of analytical grade purity unless otherwise specified.

Animal Treatment Male ICR mice (5 weeks old) were obtained from DBL Co. (Chungbuk, Korea). Animals were maintained in a $12 \mathrm{~h}$ light/dark cycle in controlled environment (temperature, $23 \pm 1^{\circ} \mathrm{C}$; humidity, $55 \%$ ), and were allowed free access to food and water. The animals were divided into 5 groups and fed either a standard diet (for negative and positive control groups) or a standard diet supplemented with various concentrations of BP $(10,20,40 \mathrm{mg} / \mathrm{kg}$ body weight (b.w.) per day) for 3 weeks. ${ }^{13)}$ After 3 weeks, the positive control group (TMT-treated group) and intervention groups were intraperitoneally injected with TMT $(2.5 \mathrm{mg} / \mathrm{kg}$ b.w.), as described elsewhere. ${ }^{14)}$ The negative control group received an injection of $0.85 \%$ saline. The behavioral tests were conducted $2 \mathrm{~d}$ after TMT injection. All experimental procedures were approved by the Animal Care and Use Committee of Korea University and performed according to animal use regulations.

Y-Maze Test Short-term spatial memory performance 

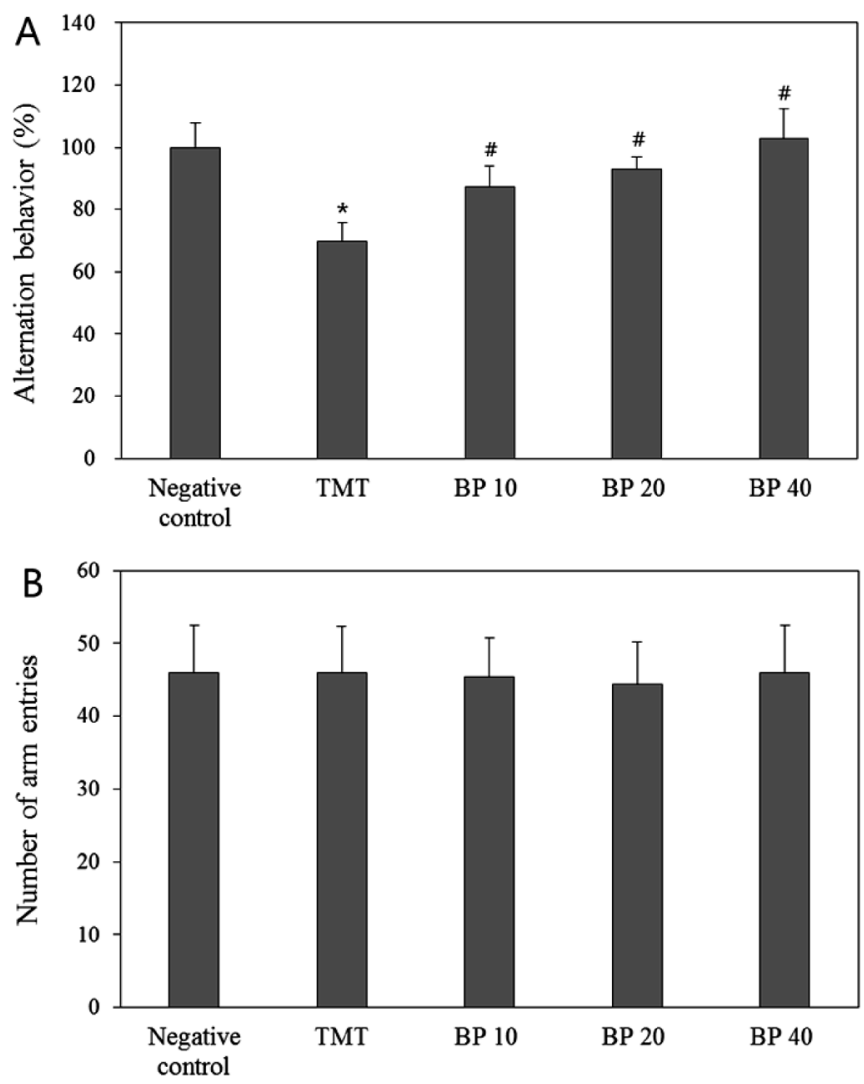

Fig. 1. Effect of BP from C. loureirii on Cognitive Impairment Induced by TMT in the Y-Maze Test

The spontaneous alternation behavior (A) and number of arm entries (B) were measured for $8 \mathrm{~min}$. The Negative control group received injections of saline. The TMT group received injections of TMT $(2.5 \mathrm{mg} / \mathrm{kg}$ b.w.). All sample groups (BP 10 , BP 20, BP 40) were injected with TMT 3 weeks after administration with BP from C. loureirii $(10,20,40 \mathrm{mg} / \mathrm{kg}$ b.w. per day). Values are mean \pm S.D. $(n=8)$. $* p<0.05$ in comparison to the control group, ${ }^{\#} p<0.05$ in comparison with the TMT group.

was tested as previously reported by Kim et $a l^{7)}$ Each mouse was placed at the end of one arm and allowed to explore freely through the maze over an 8-min session. The sequence and numbers of arm entries visited were manually recorded. The spontaneous alternation behavior (\%) was defined as the ratio of actual alternations to possible alternations (the total number of arm entries minus two), multiplied by 100 .

Passive Avoidance Test This test was carried out in a chamber with two compartments (illuminated/dark) with an electrifiable grid floor. For the acquisition trial, each mouse was placed in the illuminated compartment. Upon entry into the dark compartment, an electric foot shock $(0.5 \mathrm{~mA}, 1 \mathrm{~s})$ was applied through the grid floor. For the retention trial $24 \mathrm{~h}$ later, each mouse was again placed in the illuminated compartment. The time required to enter the dark compartment was measured (maximum $300 \mathrm{~s}$ ). ${ }^{15}$ )

Preparation of the Brain Homogenate Upon completion of the behavioral tests, the brain tissues were rapidly removed and stored at $-80^{\circ} \mathrm{C}$ until use. The tissues were homogenized with 10 volumes of phosphate buffered saline and centrifuged twice at $10000 \times \boldsymbol{g}$ for $10 \mathrm{~s}$, with a $30 \mathrm{~s}$ interval, at $4^{\circ} \mathrm{C}$. The supernatant was collected and the protein concentration was determined using the Bradford assay.

Determination of AChE Activity The rate of hydrolysis by AChE was measured using a modification of the method of Ellman et al. ${ }^{16)}$ In brief, the brain homogenates were incubated

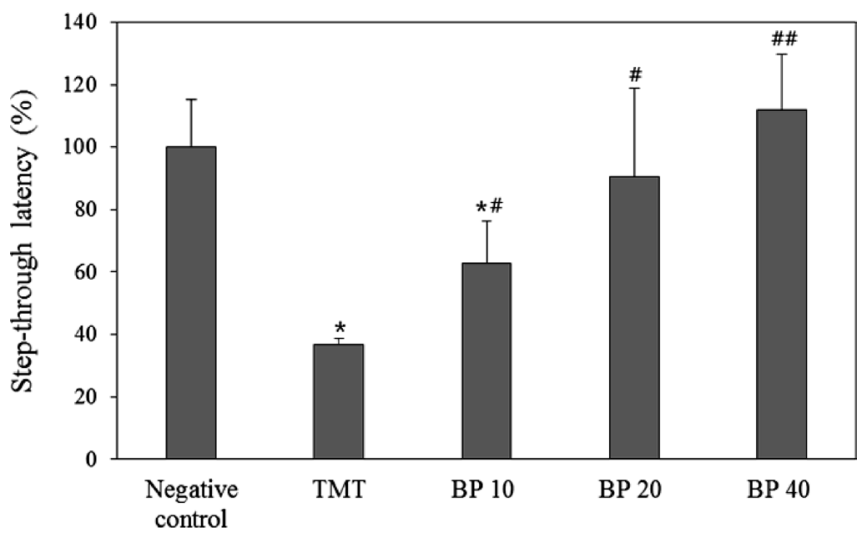

Fig. 2. Effect of BP from C. loureirii on Cognitive Impairment Induced by TMT in the Passive Avoidance Test

The step-through latency was measured over $5 \mathrm{~min}$. The Negative control group received an injection of saline. The TMT group was injected with TMT $(2.5 \mathrm{mg} / \mathrm{kg}$ bw). All sample groups (BP 10, BP 20, BP 40) were injected with TMT 3 weeks after treatment with BP from $C$. loureirii $(10,20,40 \mathrm{mg} / \mathrm{kg}$ b.w. per day). Values are mean \pm S.D. $(n=8) .{ }^{*} p<0.05$ in comparison with the control group; ${ }^{*} p<0.05$, ${ }^{\# \#} p<0.01$ in comparison with the TMT group.

in a reaction mixture $(50 \mathrm{~mm}$ sodium phosphate buffer, $\mathrm{pH} 8.0$, containing $0.5 \mathrm{~mm}$ acetylthiocholine iodide and $1 \mathrm{~mm}$ DTNB) at $37^{\circ} \mathrm{C}$ for $15 \mathrm{~min}$. Then, the absorbance was measured at $405 \mathrm{~nm}$.

Determination of Brain ACh Concentration The ACh level in brain tissue was measured using a modification of the method of Hestrin. ${ }^{17)}$ The homogenate was mixed with alkaline hydroxylamine reagent $(2 \mathrm{M}$ hydroxylamine hydrochloride containing $3.5 \mathrm{~N}$ sodium hydroxide) kept for $3 \mathrm{~h}$ at room temperature. After $1 \mathrm{~min}, 3.9 \mathrm{~N}$ hydrochloric acid and $0.37 \mathrm{M}$ ferric chloride were gradually added to the mixture, which was then centrifuged at $1500 \mathrm{rpm}$ for $15 \mathrm{~min}$. The absorbance of the supernatant was measured at $540 \mathrm{~nm}$.

Determination of Lipid Peroxidation The level of lipid peroxidation was analyzed by measuring thiobarbituric acid reactive substance (TBARS) using slightly modified method of Uchiyama and Mihara. ${ }^{18)}$ The brain homogenate was mixed with $1 \%(\mathrm{v} / \mathrm{v})$ phosphoric acid and $0.67 \%(\mathrm{v} / \mathrm{v})$ thiobarbituric acid, and the mixture was heated for $45 \mathrm{~min}$ in a boiling water bath. After cooling, the supernatant was extracted with $n$ butanol and the absorbance was measured at $532 \mathrm{~nm}$.

Statistical Analysis All results were presented as the mean \pm standard deviation (S.D.). Statistical analyses were performed using the Statistical Analysis System software package (SAS 9.4; Cary, NC, U.S.A.). Significant differences between groups were examined by one-way ANOVA followed by a Scheffe's multiple range test. A $p$ value less than 0.05 was considered statistically significant.

\section{RESULTS}

Effects of BP on TMT-Induced Memory Injury Administration of TMT resulted in a significantly lower spontaneous alternation percentage in comparison with the negative control mice (Fig. 1A). Pretreatment with BP for 3 weeks significantly increased alternation behavior in comparison with the TMTtreated group (i.e., TMT group), which indicates reversal of TMT-induced amnesia. Especially, the highest dose of BP group (i.e., BP 40 group) showed almost comparable scores in the alternation behavior (102.73\%) compared to those of 
the TMT group $(69.91 \%)$. In contrast, there were no significant differences in the numbers of arm entries among the five groups (Fig. 1B)

The passive avoidance test was used to assess short-term memory loss. As shown in Fig. 2, mice treated with TMT showed a significant decrease in the step-through latency in comparison with negative control group. BP administration reversed the cognitive deficit induced by TMT. In particular, the BP 40 group showed significantly more attenuation of step-through latency $(111.84 \%)$ than the TMT group $(36.84 \%)$.

Effects of BP on Biochemical Parameters Given the important roles of cholinergic system in cognitive functions, we examined AChE inhibitory activity and ACh content in brain tissue of TMT-treated mice. As shown in Table 1, the TMT administration increased AChE activity compared with the saline treated negative control group; in contrast, administration of BP reversed the increased AChE activity compared to those of mice treated with TMT only. In addition, it was demonstrated that ACh concentration in brain tissue of the TMT group was lower than the negative control group. The highest dose of BP (i.e., $40 \mathrm{mg} / \mathrm{kg}$ b.w.) significantly increased ACh levels compared to the levels in the TMT group. We also investigated the levels of MDA, an indicator of lipid peroxidation, in brain tissue was investigated to confirm whether BP could protect TMT-induced lipid peroxidation. The TMTtreated mice showed slightly elevated MDA levels in comparison with the negative control group, while pretreatment with $\mathrm{BP}$ at 20 and $40 \mathrm{mg} / \mathrm{kg}$ inhibited lipid peroxidation in mice brain tissue.

\section{DISCUSSION}

According to the cholinergic hypothesis, neurotransmitter deficits in the brain are one of major neuropathological features and are correlated with cognitive impairment. ${ }^{19)}$ Previously, we demonstrated inhibitory potential of $C$. loureirii extract against $\mathrm{AChE}$ in vitro, and determined that $\mathrm{BP}$, isolated from $C$. loureirii, might be a responsible AChE inhibitor. ${ }^{7)}$ In the present study, we attempted to evaluate the neuroprotective effects of BP against cognitive decline, synapse loss, and oxidative damage resulting from TMT in vivo.

TMT is an organotin compound that produces neuronal degeneration in the hippocampus, and results in behavioral alternations including hyperactivity, aggression, and cognitive impairment. ${ }^{20)}$ It has been widely used as a neurotoxin given their impacts on in vivo behavioral changes and biochemical defects in brains. ${ }^{21,22)}$ As demonstrated in many other previous studies, we were also able to confirm that TMT exposure impaired the learning and memory ability of mice, which was described by decreased spontaneous alternation behavior in the Y-maze test and reduced memory retention in the passive avoidance test. In contrast, in both in vivo behavioral tests, BP administration improved cognitive impairment induced by TMT exposure. Furthermore, there were no significant differences in body and brain weight gain among the groups, with a survival rate of $100 \%$ (data not shown).

Alternations in learning and memory might be associated with the activity of AChE in the brain. Studies have demonstrated that TMT exposure enhanced AChE staining in the hippocampal dentate gyrus, ${ }^{23)}$ and administration of TMT in mice resulted in a significant increase in AChE activity $^{24,25)}$; such effects were observed in our study as well. We demonstrated that BP regulated the TMT-induced AChE overactivity in brain tissues. Compared with the TMT group, $\mathrm{BP}$ administration decreased AChE activity in all BP groups and increased ACh levels (BP 40 group). Taken together, it is possible that increase in AChE activity in TMT-treated mice results memory impairment while BP administration improves such symptoms through inhibiting AChE activity to restore ACh levels in brain tissues.

MDA, the degradation product of the oxygen-derived free radicals and lipid peroxidation, is a predictor of the levels of oxidative membrane damage. ${ }^{26)}$ It was demonstrated that TMT increases the production of MDA (i.e., reactive oxygen species) thereby damage brain tissues in mice. ${ }^{27,28)}$ In this study, as expected, TMT treatment significantly increased MDA level in the brain tissue, compared to those in the negative control mice; in contrast, supplementation of BP significantly decreased MDA levels, compared to the TMT group. Its anti-oxidative capacity has been reported that BP from sweet potato protects PC12 cell lines and mice against amyloid beta peptide-mediated oxidative stress. ${ }^{13)}$ As the previous reports showed that $\mathrm{AChEls}$ are controlled by their respective mechanisms (e.g., suicide inhibition, or lock and key transformation), ${ }^{29)}$ further study on the mechanism would be needed. In mouse fed with BP $(10 \mathrm{mg} / \mathrm{kg})$, behavior was improved, but $\mathrm{ACh}$ and MDA levels were likely to be almost same as those of mouse treated with TMT only. It supposes that there are other mechanisms to improve mouse behavior in addition to improvement of $\mathrm{ACh}$ and oxidative stress. Additional investigations might be warranted to clarify these speculations in future.

In conclusion, we demonstrated that BP improves learning and memory deficits, induced by TMT. In our subsequent $e x$ vivo experiments, it was shown that BP reduced lipid peroxidation, increased AChE activity, and restored ACh levels in brain tissues. Therefore, it seems that cognitive dysfunction in TMT treated mice was attenuated due to its anti-oxidative potency as well as alteration of cholinergic functions. Hence, BP isolated from $C$. loureirii may be a potential preventive agent for the treatment of AD. In future, it will be interesting if this agent also exert therapeutic potentials against TMT or other

Table 1. Effect of BP $(10,20,40 \mathrm{mg} / \mathrm{kg})$ on AChE Activity, ACh Level, and Lipid Peroxidation in TMT-Induced Mouse Brain

\begin{tabular}{lccccc}
\hline \hline Biochemical parameters & Negative control & TMT & TMT+BP $(10 \mathrm{mg} / \mathrm{kg})$ & TMT+BP $(20 \mathrm{mg} / \mathrm{kg})$ & TMT+BP $(40 \mathrm{mg} / \mathrm{kg})$ \\
\hline AChE activity (\%) & $100.00 \pm 3.36$ & $118.62 \pm 1.41^{*}$ & $110.25 \pm 3.40^{\#}$ & $109.68 \pm 2.64^{\#}$ & $104.37 \pm 1.69^{\# \#}$ \\
ACh $(\mu \mathrm{mol} / \mathrm{mg}$ protein) & $0.164 \pm 0.014$ & $0.128 \pm 0.008^{*}$ & $0.136 \pm 0.005^{*}$ & $0.144 \pm 0.009^{*}$ *\# & $0.156 \pm 0.005^{\#}$ \\
TBARS (nmol/mg protein) & $1.28 \pm 0.11$ & $1.74 \pm 0.16^{*}$ & $1.63 \pm 0.16^{*}$ & $1.49 \pm 0.04^{\#}$ & $1.29 \pm 0.20^{\# \#}$ \\
\hline
\end{tabular}

The negative control group received an injection of saline. The TMT group was injected with TMT (2.5 mg/kg b.w.). All sample groups were injected with TMT 3 weeks after treatment with BP from C. loureirii $\left(10,20\right.$, and $40 \mathrm{mg} / \mathrm{kg}$ b.w. per day). Values are mean \pm S.D. $(n=8)$. ${ }^{*} p<0.05$ in comparison with the control group; ${ }^{*} p<0.05$, ${ }^{\#} p<0.01$ in comparison with the TMT group. 
AD models in which disease symptoms will be initiated first and then active constituents will be administered.

Acknowledgment This research was supported by High Value-Added Food Technology Development Program, Ministry of Agriculture, Food and Rural Affairs, Republic of Korea.

Conflict of Interest The authors declare no conflict of interest.

\section{REFERENCES}

1) Campbell VA, Gowran A. Alzheimer's disease; taking the edge off with cannabinoids? Br. J. Pharmacol., 152, 655-662 (2007).

2) Hasselmo ME. The role of acetylcholine in learning and memory. Curr. Opin. Neurobiol., 16, 710-715 (2006).

3) Schliebs R, Arendt T. The cholinergic system in aging and neuronal degeneration. Behav. Brain Res., 221, 555-563 (2011).

4) Giacobini E. Cholinergic function and Alzheimer's disease. Int. J. Geriatr. Psychiatry, 18 (Suppl. 1), S1-S5 (2003).

5) Akhondzadeh S, Abbasi SH. Herbal medicine in the treatment of Alzheimer's disease. Am. J. Alzheimer's Dis. Other Demen., 21, 113-118 (2006)

6) Schulz V. Ginkgo extract or cholinesterase inhibitors in patients with dementia: what clinical trials and guidelines fail to consider. Phytomedicine, 10 (Suppl. 4), 74-79 (2003).

7) Kim CR, Choi SJ, Kwon YK, Kim JK, Kim YJ, Park GG, Shin DH. Cinnamomum loureirii extract inhibits acetylcholinesterase activity and ameliorates trimethyltin-induced cognitive dysfunction in mice. Biol. Pharm. Bull., 39, 1130-1136 (2016).

8) Rana VS, Blazquez MA. Chemical constituents of Gynura cusimbua aerial parts. J. Essent. Oil Res., 19, 21-22 (2007).

9) Xuan TD, Toyama T, Fukuta M, Khanh TD, Tawata S. Chemical interaction in the invasiveness of cogongrass (Imperata cylindrica (L.) Beauv.). J. Agric. Food Chem., 57, 9448-9453 (2009).

10) Russo A, Cardile V, De Ioannes A, Garbarino J. Effect of litreol on the viability of human cancer cells. Chem. Biol. Interact., 179, 178-184 (2009)

11) Abdullah A-SH, Mirghani MES, Jamal P. Antibacterial activity of Malaysian mango kernel. Afr. J. Biotechnol., 10, 18739-18748 (2011).

12) Rangel-Sánchez G, Castro-Mercado E, Garcia-Pineda E. Avocado roots treated with salicylic acid produce phenol-2,4-bis(1,1-dimethylethyl), a compound with antifungal activity. J. Plant Physiol., 171, 189-198 (2014).

13) Choi SJ, Kim JK, Kim HK, Harris K, Kim CJ, Park GG, Park CS, Shin DH. 2,4-Di-tert-butylphenol from sweet potato protects against oxidative stress in PC12 cells and in mice. J. Med. Food, 16, 977-983 (2013)

14) Kim CR, Choi SJ, Oh SS, Kwon YK, Lee NY, Park GG, Kim YJ, Heo HJ, Jun WJ, Park CS, Shin DH. Rubus coreanus Miquel inhib- its acetylcholinesterase activity and prevents cognitive impairment in a mouse model of dementia. J. Med. Food, 16, 785-792 (2013).

15) Heo HJ, Kim MJ, Lee JM, Choi SJ, Cho HY, Hong B, Kim HK, Kim E, Shin DH. Naringenin from Citrus junos has an inhibitory effect on acetylcholinesterase and a mitigating effect on amnesia. Dement. Geriatr. Cogn. Disord., 17, 151-157 (2004).

16) Ellman GL, Courtney KD, Andres V Jr, Feather-Stone RM. A new and rapid colorimetric determination of acetylcholinesterase activity. Biochem. Pharmacol., 7, 88-95 (1961).

17) Hestrin S. The reaction of acetylcholine and other carboxylic acid derivatives with hydroxylamine, and its analytical application. $J$. Biol. Chem., 180, 249-261 (1949).

18) Uchiyama M, Mihara $M$. Determination of malonaldehyde precursor in tissues by thiobarbituric acid test. Anal. Biochem., 86, 271-278 (1978).

19) Schliebs R, Arendt T. The cholinergic system in aging and neuronal degeneration. Behav. Brain Res., 221, 555-563 (2011).

20) Geloso MC, Corvino V, Michetti F. Trimethyltin-induced hippocampal degeneration as a tool to investigate neurodegenerative processes. Neurochem. Int., 58, 729-738 (2011).

21) Kaur S, Chhabra R, Nehru B. Ginkgo biloba extract attenuates hippocampal neuronal loss and cognitive dysfunction resulting from trimethyltin in mice. Phytomedicine, 20, 178-186 (2013).

22) Nishimura T, Schwarzer C, Furtinger S, Imai H, Kato N, Sperk G. Changes in the GABA-ergic system induced by trimethyltin application in the rat. Brain Res. Mol. Brain Res., 97, 1-6 (2001).

23) Woodruff ML, Baisden RH. Exposure to trimethyltin significantly enhances acetylcholinesterase staining in the rat dentate gyrus. Neurotoxicol. Teratol., 12, 33-39 (1990).

24) Kim JK, Choi SJ, Bae H, Kim CR, Cho HY, Kim YJ, Lim ST, Kim CJ, Kim HK, Peterson S, Shin DH. Effects of methoxsalen from Poncirus trifoliata on acetylcholinesterase and trimethyltin-induced learning and memory impairment. Biosci. Biotechnol. Biochem., 75, 1984-1989 (2011).

25) Ha JS, Jin DE, Park SK, Park CH, Seung TW, Bae DW, Kim DO, Heo HJ. Antiamnesic effect of Actinidia arguta extract intake in a mouse model of TMT-induced learning and memory dysfunction. Evid. Based Complement. Alternat. Med., 2015, 876484 (2015).

26) Barber AA, Bernheim F. Lipid peroxidation: its measurement, occurrence, and significance in animal tissues. Adv. Gerontol. Res., 2, 355-403 (1967).

27) Ali SF, LeBel CP, Bondy SC. Reactive oxygen species formation as a biomarker of methylmercury and trimethyltin neurotoxicity. $\mathrm{Neu}$ rotoxicology, 13, 637-648 (1992).

28) Shin EJ, Suh SK, Lim YK, Jhoo WK, Hjelle OP, Ottersen OP, Shin CY, Ko KH, Kim WK, Kim DS, Chun W, Ali S, Kim HC. Ascorbate attenuates trimethyltin-induced oxidative burden and neuronal degeneration in the rat hippocampus by maintaining glutathione homeostasis. Neuroscience, 133, 715-727 (2005).

29) Colovic MB, Krstic DZ, Lazarevic-Pasti TD, Bondzic AM, Vasic VM. Acetylcholinesterase inhibitors: pharmacology and toxicology. Curr. Neuropharmacol., 11, 315-335 (2013). 